\title{
High-performance Pixel-level Grasp Detection based on Adaptive Grasping and Grasp-aware Network
}

This paper was downloaded from TechRxiv (https://www.techrxiv.org).

LICENSE

CC BY 4.0

SUBMISSION DATE / POSTED DATE

$26-05-2021 / 12-10-2021$

CITATION

Dexin, Wang (2021): High-performance Pixel-level Grasp Detection based on Adaptive Grasping and Graspaware Network. TechRxiv. Preprint. https://doi.org/10.36227/techrxiv.14680455.v2

$\mathrm{DOI}$

10.36227/techrxiv.14680455.v2 


\title{
High-performance Pixel-level Grasp Detection based on Adaptive Grasping and Grasp-aware Network
}

\author{
Dexin Wang, Chunsheng Liu, Member, IEEE, Faliang Chang, Nanjun Li, and Guangxin Li
}

\begin{abstract}
Machine vision based planar grasping detection is challenging due to uncertainty about object shape, pose, size, etc. Previous methods mostly focus on predicting discrete gripper configurations, and may miss some ground-truth grasp postures. In this paper, a pixel-level grasp detection method is proposed, which uses deep neural network to predict pixel-level gripper configurations on RGB images. Firstly, a novel Oriented Arrow Representation model (OAR-model) is introduced to represent the gripper configuration of parallel-jaw and three-fingered gripper, which can partly improve the applicability to different grippers. Then, the Adaptive Grasping Attribute model (AGAmodel) is proposed to adaptively represent the grasping attribute of objects, for resolving angle conflicts in training and simplifying pixel-level labeling. Lastly, the Adaptive Feature Fusion and Grasp Aware Network (AFFGA-Net) is proposed to predict pixel-level OAR-models on RGB images. AFFGA-Net improves the robustness in unstructured scenarios by using Hybrid Atrous Spatial Pyramid and Adaptive Decoder connected in sequence. On the public Cornell dataset and actual objects, our structure achieves $99.09 \%$ and $98.0 \%$ grasp detection accuracy respectively. In over 2,400 robotic grasp trials, our structure achieves average success rate of $98.77 \%$ in single-object scenarios and $93.69 \%$ in cluttered scenarios. Moreover, AFFGA-Net completes a grasp detection pipeline within $15 \mathrm{~ms}$.
\end{abstract}

Index Terms-grasp detection, oriented arrow representation, adaptive grasping attribute model, convolutional neural network

\section{INTRODUCTION}

W ITH the advantage of high automation, robot grasping is widely used in industrial production [1] [2]. Reliable robotic grasping is challenging due to uncertainty about object shape, pose, size, etc. Recently, many algorithms have claimed to be effective for handling stacked scenarios as well as grasping novel objects [3] [4]. However, how to represent a gripper configuration and what is the output format of the learning algorithm are still open questions.

How to represent a gripper configuration? A complete gripper configuration includes 3D location, 3D orientation, gripper grasp width, etc [5]. Because predicting all variables is complicated, this task is often simplified to predict a 'representation' of the gripper configuration, which is a projection

Dexin Wang, Chunsheng Liu, Faliang Chang, Nanjun Li and Guangxin Li are with the School of Control Science and Engineering, Shandong University, Ji'nan, Shandong 250061, China (e-mail: flchang@sdu.edu.cn, liuchunsheng@sdu.edu.cn). (Corresponding author: Faliang Chang, Chunsheng Liu)

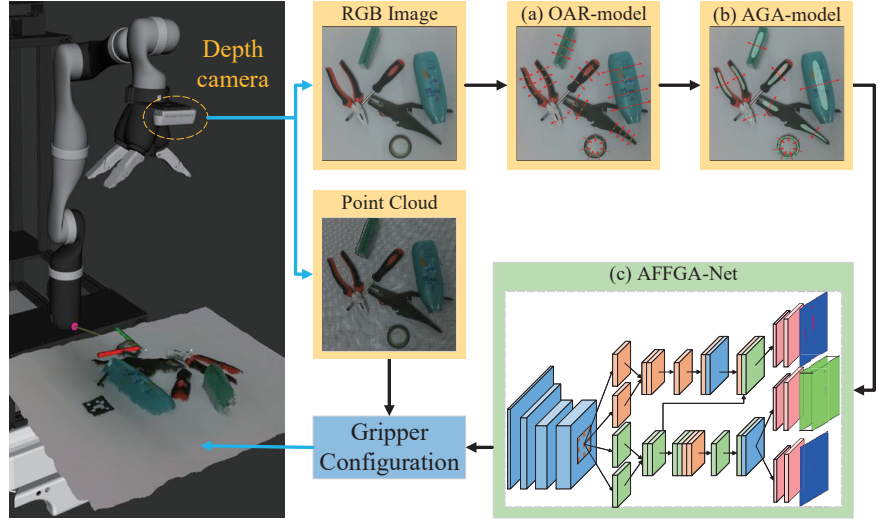

Fig. 1. Our pixel-level grasp detection method.

of the gripper configuration on a plane. Jiang et al. [6] use an oriented rectangle to represent the gripper configuration of the parallel-jaw gripper; this method sets the gripper jaw size as an empirical value, but the jaw size is not potentially related to the object size, which makes the neural network confused. Mahler et al. [7] use a point and an angle to represent the gripper configuration; this method sets the grasp width as a constant which limits the size of objects that can be grasped. Moreover, they are only applicative for the parallel-jaw gripper.

What is the output format of the learning algorithm? We argue that the output format of the learning algorithm is the grasping attribute of the object, i.e. the sum of all grasp configurations that an object can be grasped by a gripper. Most methods based on rectangle representation predict multiple discrete oriented rectangle on RGB images [8] [9]. Besides, Mahler et al. first sample some gripper configurations, and then evaluate their confidence [7] [10] [11]. However, the feasible gripper configurations on the object are continuous, and these methods may miss some ground-truth grasp postures.

To overcome these existing problems, we propose a pixellevel grasp detection method to generate pixel-level gripper configurations for parallel-jaw and three-fingered gripper, which consists of three parts including Oriented Arrow Representation model (OAR-model), Adaptive Grasping Attribute model (AGA-model) and Adaptive Feature Fusion and Grasp Aware Network (AFFGA-Net).

First, we design the OAR-model to improve the learnability and applicability of the grasp representation. By simplifying a three-fingered gripper into a parallel-jaw gripper with two jaws 
with different sizes, OAR-model is applicative for the paralleljaw gripper and the simplified three-fingered gripper. OARmodel avoids the confusion of the neural network learning by constraining the size of the gripper jaw, and is applicative for objects with different sizes by using variable grasp width.

Second, to optimize the learning process of network, we propose the AGA-model which represents the grasping attribute of objects. One pixel may correspond to multiple OARmodels with different grasp angles, which may cause angle conflicts. By combining adjacent OAR-models, AGA-model resolves angle conflicts in training and avoids the extremely complicated pixel-level labeling process.

Third, the AFFGA-Net is proposed to generate an OARmodel and confidence at every pixel on RGB images. The pixel-level mapping avoids missing ground-truth grasp postures, and overcomes limitations of current deep-learning grasping techniques including discrete sampling of grasp candidates and large computation consuming. Moreover, AFFGANet is robust for objects with various shapes and sizes, by using Hybrid Atrous Spatial Pyramid and Adaptive Decoder which can adaptively extract and decode multi-scale features.

On the Cornell Grasp dataset [12], our method achieves accuracy of $99.09 \%$ and $98.64 \%$ on image-wise and objectwise splitting respectively, and outperforms the latest stateof-the-art approach by $1.35 \%$ and $2.03 \%$, respectively. On the randomly selected household objects, our method achieves $98.0 \%$ grasp detection accuracy. In over 2,400 robotic grasp trials, our structure achieves an average success rate of $98.77 \%$ on single-object scenarios and $93.69 \%$ on cluttered scenarios. Moreover, AFFGA-Net completes a grasp detection pipeline within $15 \mathrm{~ms}$, which can be used for real-time applications. Code is provided at https://github.com/liuchunsense/AFFGANet.

The contributions of our study are summarized as follows:

1) The OAR-model is designed to represent the gripper configuration of parallel-jaw gripper and simplified threefingered gripper, which avoids the confusion of the network learning and is applicative for objects with different sizes.

2) The AGA-model is proposed to resolve angle conflicts in training and avoids the extremely complicated pixel-level labeling process.

3) The AFFGA-Net is proposed to generate pixel-level OARmodel on RGB images, which avoids missing ground-truth grasp postures and reduces computational times.

4) Our structure achieves the state-of-the-art performance on the Cornell Grasp dataset and is proved effective for novel objects in cluttered scenarios.

This paper is organized as follows. Section II discusses related grasp detection methods. Section III introduces our method in detail. Section IV introduces our experiment setup. Section V demonstrates detailed experiments and results, and then Section VI presents the conclusion.

\section{Related Work}

The goal of grasp detection is to find a proper posture using the visual information in different scenarios, and the gripper can stably grasp the target when closing the jaws in this posture. The methods can be roughly divided into two categories: analytic methods and empirical methods [13]. Analytic methods use mathematical and physical models of geometry, kinematics and dynamics to calculate stable grasping parameters [14] [15]; yet, they usually can not transfer well to the real world due to the difficultly in modelling physical interactions between a manipulator and an object [16]. In contrast, empirical methods do not require object 3D models; they train the grasp model using known objects and use this model to detect the grasping posture of unknown objects [17] [18] [19]. In recent years, some deep learning based methods have designed to first detect planar grasp representation and then map the representation to grasp posture in the world coordinate system; these methods usually perform better than traditional empirical methods based on shape primitives [20] [21], machine learning [22], etc.

Grasping representation. A planar grasp representation generally includes grasp point, grasp angle, and grasp width as least. Saxena et al. [23] use supervised learning to predict a grasp point from the image and successfully extend it to new targets. Le et al. [24] suggest that a pair of points are used to represent grasping. Jiang et al. [6] reduce the dimensionality of the 7-dimensional gripper configuration (the 3D location, the $3 \mathrm{D}$ orientation and the distance between the two fingers) in the real environment to obtain a simplified five-dimensional rectangle grasping representation. These representations suffer from the problem that they are limited to parallel plate gripper.

Network. Previous grasp detection networks are often based on object detection network [25]. Zhou et al. [26] use ResNet50 as the feature extractor and adopt anchor mechanism [27] to predict the five-dimensional rectangle grasp model, which greatly improves the prediction accuracy. Asif et al. [28] overcome limitations of individual models by combining CNN structures with feature fusion and producing grasping with confidence scores at different levels of the image hierarchy (i.e. global-, region-, and pixel-levels). However, these algorithms cannot generate dense rectangles, which makes it hard to accurately predict the grasping attribute of the object.

\section{OUR METHOD}

To overcome the limitations of previous grasp detection methods that may miss some ground-truth grasp postures, we propose a pixel-level grasp detection method with three main parts, which is shown in Fig. 1. Firstly, the Oriented Arrow Representation model (OAR-model) is introduced to represent mapping of the gripper configuration on plane. Secondly, all OAR-models on the object are merged into multiple Adaptive Grasping Attribute models (AGA-models), and the AGAmodels are labeled as targets to train the Adaptive Feature Fusion and Grasp Aware Network (AFFGA-Net). AFFGA-Net inputs a RGB image and outputs an OAR-model at every pixel. Then, the gripper configuration is calculated using the optimal OAR-model and point cloud. Lastly, the robot approaches the target and closes the jaws.

\section{A. Oriented Arrow Representation Model}

Unlike general multi-fingered grippers, each finger of the three-fingered gripper (Fig. 2(a)) can only apply force toward 


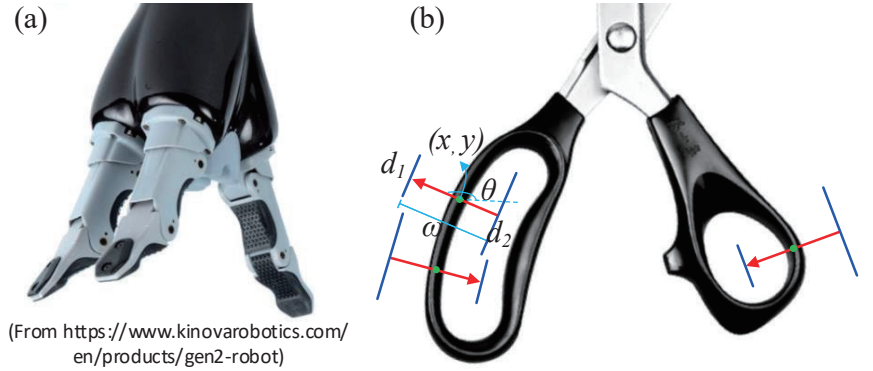

Fig. 2. (a): The three-fingered gripper. (b): Examples of OAR-models.
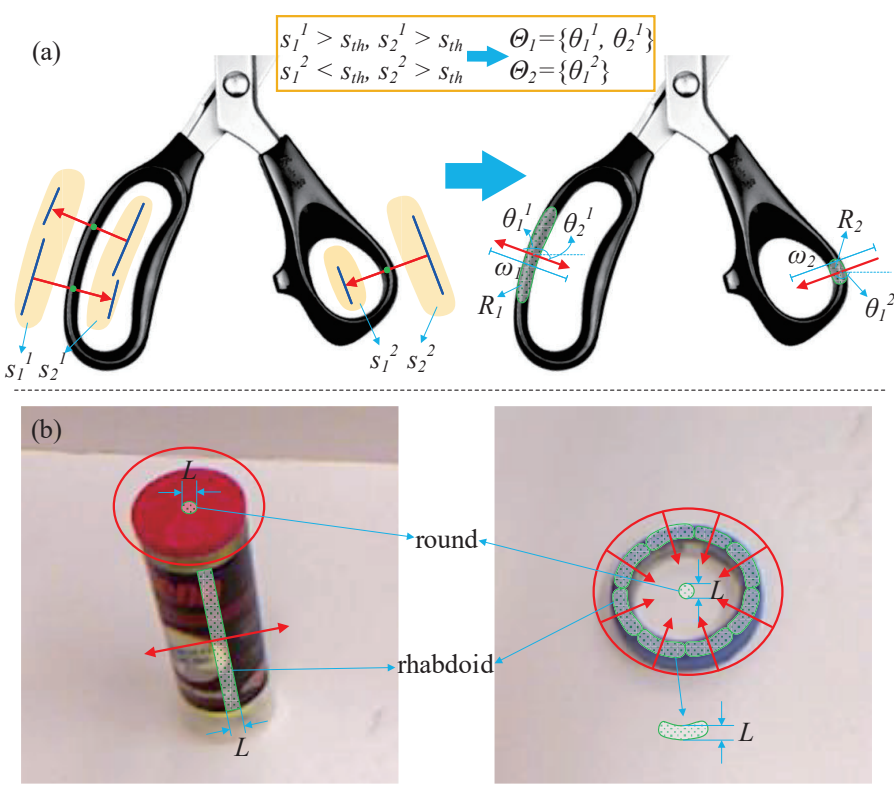

Fig. 3. (a): Illustration of AGA-models. An AGA-model is defined by the grasp region $R$ (green pattern), grasp angle $\Theta$ (directions of the red arrow), and grasp width $\omega$ (length of the red arrow). The superscript of $\theta$ and $s$ is used to distinguish different AGA-models. (b): Examples of rhabdoid and round graspable parts and the AGA-models in the graspable parts. The red circle indicates that $\Theta=\{\theta \mid 0 \leq \theta<2 \pi\}$. The diameter of the circle represents the grasp width of the AGA-model.

the center of the gripper. In order to use only one grasp representation to represent both parallel-jaw gripper and threefingered gripper. We simplify the three-fingered gripper into a parallel-jaw gripper with two jaws of various sizes by keeping two adjacent fingers moving in sync. The OAR-model is shown in Fig. 2(b) and represented as:

$$
G_{r}=\left\{x, y, \omega, d_{1}, d_{2}, \theta\right\}
$$

where $(x, y)$ denotes the grasp point, $\omega$ is the grasp width, $d_{1}$ and $d_{2}$ represent the size of two jaws respectively $\left(d_{1}<d_{2}\right)$, and $\theta$ is the grasp angle.

Given the OAR-model and the point cloud of object, the $3 \mathrm{D}$ coordinate of the grasp point is the projection point of $(x, y)$ in the point cloud. For three-fingered gripper, the $d_{1}$ position is where the single finger places, and the $d_{2}$ position is where the other two fingers place. For parallel-jaw gripper, two fingers are placed in the $d_{1}$ or $d_{2}$ position respectively. The gripper is perpendicular to the table, and all fingers apply forces towards the grasping point to grasp object.
Rectangle representation sets the size of the gripper jaw as an empirical value, but the jaw size is not potentially related to the object size, which makes the neural network confused. We set $d_{1}$ and $d_{2}$ as the mapping of the real size of the threefingered gripper jaw in the image coordinate system. This constraint avoids the confusion of training a network. Thus, the network can focus on learning the mapping from the object image to other values of the OAR-model. Compared with DexNet 2.0 [7], the grasp width of OAR-model is variable and can be learned to fit objects with different sizes.

\section{B. Adaptive Grasping Attribute Model}

There are two difficulties with learning pixel-level mapping: (1) One pixel may correspond to multiple OAR-models with different grasp angles, which may cause angle conflicts. (2) It is extremely time-consuming to label pixel-level OARmodels. In order to solve the above problems, we propose AGA-model to model the grasping attribute of the object, which is composed of multiple adjacent OAR-models. AGAmodel transforms single-angle learning tasks into multi-angle learning tasks, and transforms pixel-level labeling tasks into region-level labeling tasks.

The AGA-model is shown in Fig. 3(a) and represented as:

$$
G_{m}=\{R, \Theta, \omega\}
$$

where $R$ represents grasp region, $\Theta$ represents grasp angle, and $\omega$ represents grasp width.

1) grasp region: The points on the object that can be grasped are clustered into multiple regions. The defined grasp region is composed of multiple adjacent grasp points $(x, y)$ on an object, in which these grasp points have the same grasp angle $\theta$ and grasp width $\omega$. The shape of the grasp region is similar to that of the graspable part of object. To avoid moving the object while grasping, the grasp point is located near the central axis of the object. We limit the maximum width (perpendicular to the central axis of object) of the grasp region to $L$ ( $1 \mathrm{~cm}$ in this study). For the finer graspable part with a width less than $L$, the edge of the grasp region is aligned with the graspable part. For the thicker graspable part with a width greater than $L$, the grasp region is located inside the graspable part (Fig. 3(b)).

2) grasp angle: The parallel-jaw gripper may grasp objects symmetrically when the space around the object can accommodate the gripper jaw. Comparing with parallel-jaw gripper, the three-fingered gripper needs to additionally consider the different space around objects, such as tape with a small inner ring, scissor handle, etc. Formally, let $S$ represent the shape of the graspable part of the object. If $S$ is rhabdoid, let $s_{1}$ and $s_{2}$ denote the space on two sides of the graspable part, respectively. We set the elements of $\Theta$ according to the following three situations:

- When $S$ is rhabdoid, if $\max \left(s_{1}, s_{2}\right)$ is greater than $s_{t h}$ and $\min \left(s_{1}, s_{2}\right)$ is less than $s_{t h}, \Theta$ contains only one grasp angle:

$$
\Theta=\left\{\theta_{1}\right\}, \text { if } \max \left(s_{1}, s_{2}\right) \geq s_{t h}, \min \left(s_{1}, s_{2}\right)<s_{t h}
$$


(a) (b)

(c)

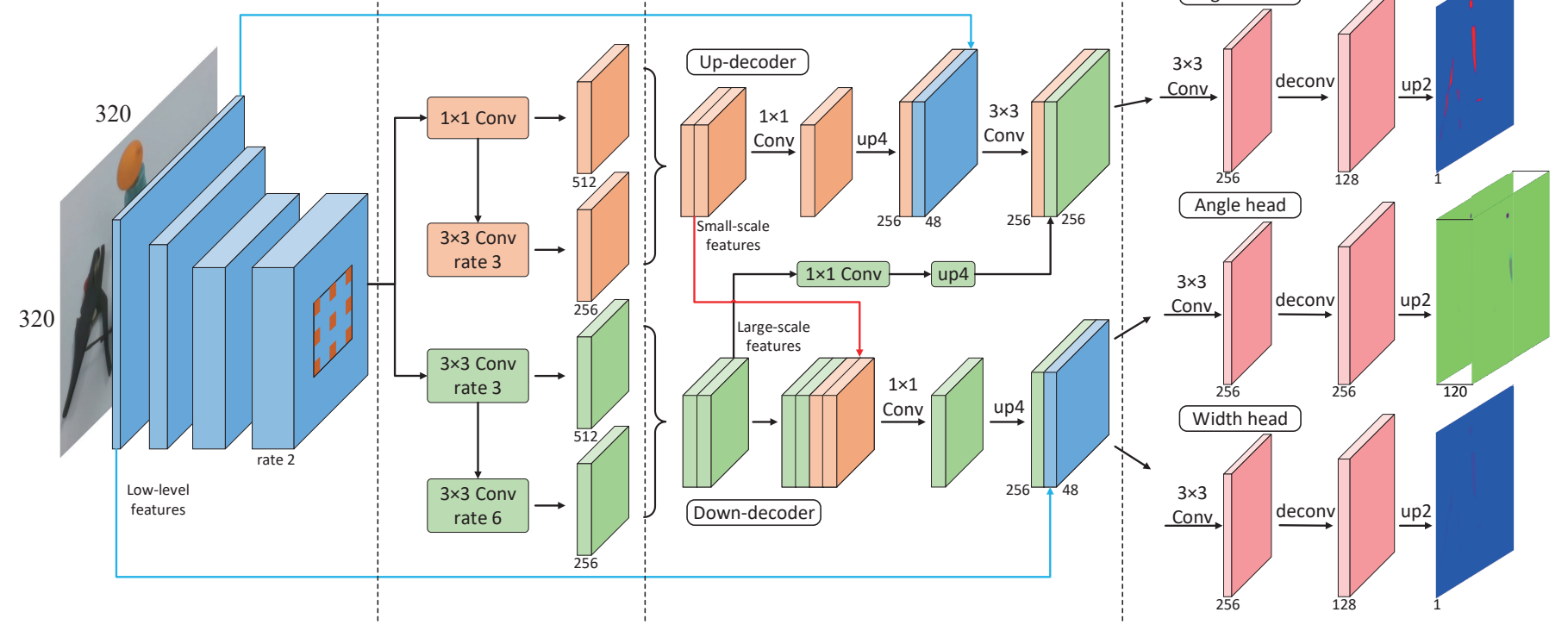

Fig. 4. Structure of the AFFGA-Net. (a): Shared Encoder. (b): Hybrid Atrous Spatial Pyramid. (c): Adaptive Decoder. (d): Grasping Heads.

where $\theta_{1}$ points to the small side of the graspable part.

- When $S$ is rhabdoid, if $\min \left(s_{1}, s_{2}\right)$ is greater than $s_{t h}$, $\Theta$ contains two retrorse grasp angles:

$$
\Theta=\left\{\theta_{1}, \theta_{2}\right\}=\left\{\theta_{1}, \theta_{1}+\pi\right\} \text {, if } \min \left(s_{1}, s_{2}\right) \geq s_{t h}
$$

where $\theta_{1}$ and $\theta_{2}$ point to the two sides of the graspable part respectively.

- When $S$ is round, $\Theta$ contains all values between 0 and $2 \pi$ :

$$
\Theta=\{\theta \mid 0 \leq \theta<2 \pi\} \text {, if } S=\text { round }
$$

We set $s_{t h}=d_{2}$ to avoid collision between the gripper and the object. Examples of rhabdoid and round graspable part and the AGA-model in the graspable part are shown in Fig. 3(b).

3) grasp width: The grasp width is an integer value whose definition and labeling method are the same as [6].

Taking any point in the grasp region $R$ as the grasp point $(x, y)$, and selecting any element in $\Theta$ as the grasp angle $\theta$, an OAR-model is built with the grasp width $\omega$. AGA-model merges the grasp angles on the OAR-models located at the same location into a set to avoid angle conflicts. Besides, the neural network can be trained by labeling AGA-model on the dataset, which greatly simplifies the labeling process.

\section{Adaptive Feature Fusion and Grasp Aware Network}

Based on the OAR-model and AGA-model, we take the planar grasp detection problem as a pixel-level segmentation problem, and propose a novel AFFGA-Net to fast generate an optimal gripper configuration to guide robot grasping.

Based on deeplabv3+ [29], we propose four solutions to build our AFFFGA-Net (Fig. 4): (1) Hybrid Atrous Spatial Pyramid (HASP); (2) Adaptive Decoder (AD); (3) Mixed Upsampling; (4) Sigmoid. We introduce the baseline and our solutions separately as follow.
1) Baseline: We retain all the details of the encoderdecoder structure of deeplabv3+ [29], and only modify the final task head to output pixel-level OAR-models. ResNet101 [30] is utilized as the backbone of Shared Encoder. Three semantic grasping heads are designed and parallelly attached to the decoder, including region head, angle head and width head, whose output channels are $\{1, K, 1\}$ ( $K=120$ in this study). Region head outputs the confidences that each pixel point is located in the grasp region $R$. Angle head outputs the category $k$ of the grasp angle corresponding to each point, from which we calculate the grasp angle by $\theta=\frac{2 \pi}{K} k$. Width head outputs the grasp width corresponding to each point. The point with the maximal confidence is chosen as the grasp point $(x, y)$, and the grasp angle $\theta$ and grasp width $\omega$ are the predicted results at the $(x, y)$ position. The optimal OARmodel is built with the grasp point $(x, y)$, grasp angle $\theta$ and grasp width $\omega$. AFFGA-Net decomposes the grasp detection problem into three sub-problems: grasp region segmentation, grasp angle classification and grasp width regression.

2) Hybrid Atrous Spatial Pyramid: Chen et al. [31] verify that Atrous Spatial Pyramid Pooling (ASPP) is effective for improving the segmentation accuracy of multi-scale objects. Nonetheless, the large hole rate (rate $=\{6,12,18\}$ ) leads to low information utilization and loss of local features. We design HASP to solve the above problems.

HASP includes two parallel two-layer feature pyramids, which are used to extract features with different scales, and each pyramid consists of two atrous convolutions connected in series. The cascade structure improves the information utilization rate without reducing the receptive field, and the parallel structure avoids the redundancy between multi-scale features. The details of HASP are shown in Fig. 4(b).

3) Adaptive Decoder: In the AGA-model, the grasp region of the thicker graspable part is located inside, and the grasp region of the finer graspable part is aligned with the edge. 
Therefore, we design Adaptive Decoder (AD) to avoid the predicted grasp region of thicker objects close to the edge, which is shown in Fig. 4(c).

AD includes two parallel decoder networks, which merge different levels of features in different orders. In the updecoder, the small-scale features are first concatenated with the low-level features from the backbone, then concatenated with large-scale features. In the down-decoder, the large-scale features are first concatenated with the small-scale features, then concatenated with the low-level features. The difference in feature fusion order makes the output of the upper decoder not contain the edge information of large-scale objects, while the output of the lower decoder contains all the information of objects with different scales. The features output by the upper decoder are input into the region head to predict the grasp region. The features output by the lower decoder are used to predict the grasp angle and grasp width, because the grasp angle and grasp width on objects with different scales are all related to accurate edge information.

4) Mixed Upsampling: The decoder features are computed with output stride $=4$. Since the grasp region is smaller than the object mask, we use a $3 \times 3$ deconvolution followed by a bilinear upsampling to accurately restore the grasp region.

5) Sigmoid: Predicting grasp angle is a multi-label singleclassification task. We use sigmoid function to normalize the output of angle head to avoid competition among categories.

\section{Loss Function}

We calculate the loss separately for the output of each head and use the sum of losses to optimize AFFGA-Net.

1) grasp region: Predicting grasp region is a binary classification problem. We first use the sigmoid function to normalize the prediction results followed by binary cross-entropy function (BCE) to calculate the loss, which is defined as:

$$
L_{r e g}=-\frac{1}{N} \sum_{n=0}^{N}\left[y_{q}^{n} \cdot \log \left(p_{q}^{n}\right)+\left(1-y_{q}^{n}\right) \cdot \log \left(1-p_{g}^{n}\right)\right]
$$

where $N$ is the size of output feature maps, $p_{q}^{n}$ is the predicted probability at the $n$ position, $y_{q}^{n}$ is the corresponding label.

2) grasp angle: After using the sigmoid function to normalize the output of angle head, the BCE function is utilized to calculate the grasp angle loss, which is defined as:

$L_{\text {ang }}=-\frac{1}{N \times L} \sum_{n=0}^{N} \sum_{l=0}^{L}\left[y_{l}^{n} \cdot \log \left(p_{l}^{n}\right)+\left(1-y_{l}^{n}\right) \cdot \log \left(1-p_{l}^{n}\right)\right]$

where $p_{l}^{n}$ represents the probability that the predicted grasp angle is within $\left[\frac{l}{L} \times 2 \pi, \frac{l+1}{L} \times 2 \pi\right]$ at the $n$ position, $y_{l}^{n}$ is the corresponding label. We show in Sec.V-A that using sigmoid instead of softmax increases accuracy.

3) grasp width: Predicting the grasp width is a regression problem. We use the BCE function to calculate the loss of grasp width branch:

$$
L_{w i d}=-\frac{1}{N} \sum_{n=0}^{N}\left[y_{w}^{n} \cdot \log \left(p_{w}^{n}\right)+\left(1-y_{w}^{n}\right) \cdot \log \left(1-p_{w}^{n}\right)\right]
$$

where $p_{w}^{n}$ is the predicted grasp width at the $n$ position, $y_{w}^{n}$ is the corresponding label.
4) multi-task loss: In order to balance the loss of each branch, the final multi-task loss is defined as:

$$
L_{\text {total }}=\gamma_{1} \times L_{\text {reg }}+\gamma_{2} \times L_{\text {ang }}+\gamma_{3} \times L_{w i d}
$$

where $\gamma_{1}, \gamma_{2}$ and $\gamma_{3}$ are weight coefficients of the loss. In our study, we experimentally set $\gamma_{1}=1, \gamma_{2}=10$ and $\gamma_{3}=5$.

\section{EXPERIMENTS SET-UP}

The performance of the proposed method is evaluated on the Cornell Grasp dataset [12] and a test set captured in actual scenarios. The grasp detection accuracy and robotic grasp success rate are selected as the main performance metric. We also propose a simplified method to label AGA-model for training AFFGA-Net.

\section{A. Training Dataset}

To train and test AFFGA-Net, we create two datasets:

1) Cornell dataset: There are 878 images in the Cornell dataset which contains 240 graspable objects [12]. We relabel the images with AGA-model.

2) Clutter dataset: Because there is no public available dataset for cluttered scenarios on RGB images, a Clutter dataset is built by our lab and publicly released ${ }^{1}$. There are 505 images in the dataset which contains 80 graspable objects shown in Fig. 5. Each image contains 1 to 10 stacked objects.

The labeling method is described as follows. The shape of the graspable part of the object is approximately round or rhabdoid. We simplify the grasp region into a standard figure based on the shape of the graspable part.

1) When the shape of the graspable part is round, the grasp region $R$ is approximately circular in the middle of the graspable part. We label it as a circle whose center coincides with the center of the graspable part, and the grasp angle $\Theta$ contains all values from 0 to $2 \pi$.

2) When the shape of the graspable part is rhabdoid, the grasp region $R$ is approximately rectangular in the middle of the graspable part. We label it as a rotatable rectangle which is symmetrical along the central axis of the graspable part. The grasp angle $\Theta$ has one or two elements according to the size of space on both sides of the graspable part.

The labelling method of the grasp angle $\Theta$ and grasp width $\omega$ is described in Section III-B.

In Cornell dataset, we randomly select $75 \%$ as the training set and the remaining $25 \%$ as the test set. The evaluation methods are divided into the following two principles:

1) Image-wise splitting divides the images into the train set and the test set at random. This aims to test the generalization ability of the network on new positions and orientations of an trained object.

2) Object-wise splitting divides the dataset at the object instance level. All images of an instance are put into the same set. This aims to test the generalization ability of the network to new objects.

${ }^{1}$ https://github.com/liuchunsense/Clutter-Grasp-Dataset 


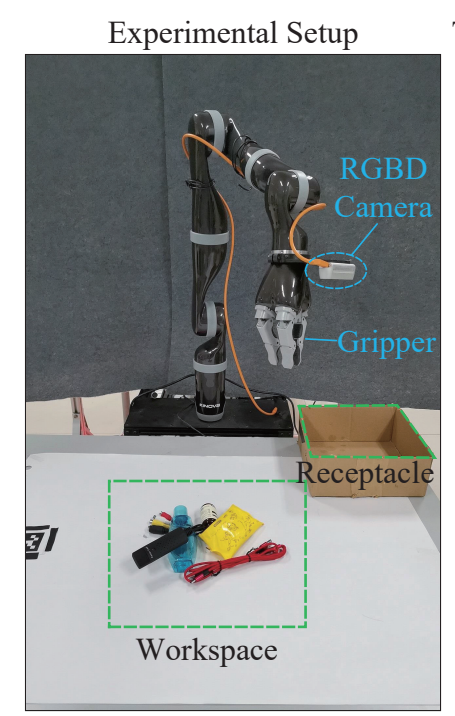

Training Objects (Clutter Dataset)

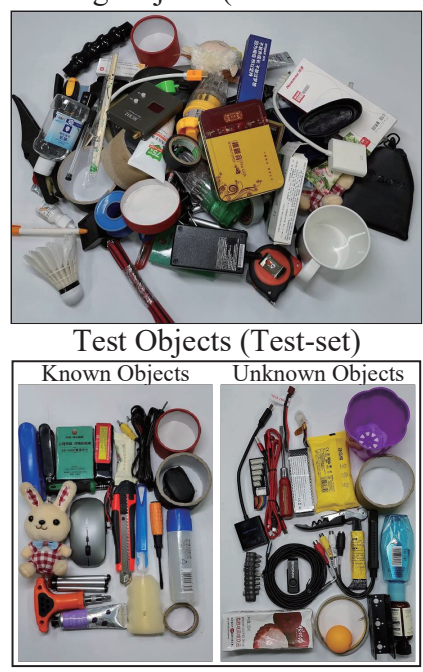

Fig. 5. Left: Set-up for robotic grasping experiments. For each experiment, $M$ objects from the Test-set are randomly dropped on the center of the workspace, at which point the robot iteratively plans grasps from RGB images and attempts to lift and transport the objects to a receptacle. Top-Right: A set of 80 training objects in Clutter dataset with various shapes, sizes, and material properties. Bottom-Right: A set of 40 test objects in Test-set. Half of the objects appear in Clutter dataset (known objects), and the other half are novel objects (unknown objects).

\section{B. Training Details}

In order to facilitate the training of AFFGA-Net, we normalize the labelled data as follows:

Grasp Confidence. We treat grasp confidence $Q$ of each pixel point as a binary label, and set the already labelled grasp points in grasp region a value of 1 . All other points are 0 .

Grasp Angle. Each grasp angle $\theta$ in set $\Theta$ is labelled in the range $[0,2 \pi)$. We discretize $\theta$ to $k=\left\lfloor\frac{120}{2 \pi} \theta\right\rfloor, k \in[0,119]$.

Grasp Width. We scale the values of $\omega$ by $\frac{1}{W}$ to put it in the range $[0,1]$. We set $W=400$ to prevent the label of the grasp width from exceeding 1 during data enhancement.

We perform data enhancement in multiple ways. We take a centre crop of $320 \times 320$ pixels with random translation up to 30 pixels horizontally and vertically. This image patch is then randomly rotated up to 30 degrees in both clock-direction and anticlock-direction. Then the image is randomly flipped horizontally. After that, we put the image into AFFGA-Net.

AFFGA-Net is implemented with PyTorch. We use Adam optimizer to optimize the networks. The initial learning rate is set to 0.001 . The network is trained end-to-end for 500 epochs and learning-rate decays at a rate of 0.5 times in the range $[100,200,300,400]$ of epochs.

\section{Evaluation Metrics for Grasp Detection}

The predicted grasping is correct when the following two conditions are met:

1) The difference between the predicted grasp angle $\theta$ and the labelled grasp angle is less than $30^{\circ}$.

2) The Jaccard index of the predicted grasp and the label is higher than $25 \%$. The Jaccard index for a predicted grasp $G$ and a labelled grasp $G^{*}$ is defined as:
TABLE I

ABLATION EXPERIMENT.

\begin{tabular}{ccccc|c|c}
\hline Baseline & Mix. up & Sigmoid & HASP & AD & Img (\%) & Obj (\%) \\
\hline$\checkmark$ & & & & & 94.98 & 95.45 \\
$\checkmark$ & $\checkmark$ & & & & 96.80 & 96.36 \\
$\checkmark$ & $\checkmark$ & $\checkmark$ & & & 98.17 & 97.27 \\
$\checkmark$ & $\checkmark$ & $\checkmark$ & $\checkmark$ & & 98.17 & 98.18 \\
$\checkmark$ & $\checkmark$ & $\checkmark$ & $\checkmark$ & $\checkmark$ & 99.09 & 98.64 \\
\hline
\end{tabular}

"Mix. up: Mixed Upsampling. Sigmoid: Sigmoid normalization for angle head. HASP: Hybrid Atrous Spatial Pyramid. AD: Adaptive Decoder. Img: Accuracy in image-wise splitting. Obj: Accuracy in object-wise splitting.

$$
J\left(G^{*}, G\right)=\frac{\left|G^{*} \cap G\right|}{\left|G^{*} \cup G\right|}
$$

To compare with methods based on rectangle representation, we set both $d_{1}$ and $d_{2}$ of OAR-model to 30 pixels during the testing phase, which is approximately the mean value of the labeled gripper jaw sizes in the Cornell dataset [12].

\section{Test Objects}

We build a set of objects on which we test the grasp detection accuracy and robotic grasp success rate (Fig. 5).

Test-set. The set consists of 40 household objects with varying sizes, shapes and difficulties. Half of the objects appear in Clutter dataset. The weight of all objects is less than 200 grams to ensure that the robot can grasp the objects when the grasp point deviates from the object's center of gravity.

\section{E. Physical Set-up}

To perform robotic grasping experiments, we use a Kinova Gen2 7DOF robot fitted with a Kinova KG-3 gripper. Our camera is an Intel RealSense D435i RGB-D camera and is mounted to the wrist of the robot. This set-up is shown in Fig. 5. The AFFGA-Net is performed on a PC running Ubuntu 16.04 with a $3.5 \mathrm{GHz}$ Intel Core i9-9900 CPU and NVIDIA TITAN-XP graphics card.

\section{EXPERIMENTS}

\section{A. Ablation Experiment}

In this subsection, we perform an ablation study to evaluate the impact of each component of the proposed AFFGA-Net on performance. Table. I summarizes the experimental results.

All networks are trained and tested in Cornell dataset. The baseline gets accuracy of $94.98 \%$ and $95.45 \%$ in imageand object-wise splitting, respectively. Mixed Upsampling increases accuracy by $1.8 \%$ and $0.9 \%$, because deconvolution reduces the loss of detail during the upsampling process. Sigmoid function increases accuracy by $1.4 \%$ and $0.9 \%$ due to avoiding competition between each category of grasp angle. HASP increases accuracy by $0.9 \%$ in object-wise splitting, and $\mathrm{AD}$ increases accuracy by $0.9 \%$ and $0.4 \%$ by extracting and decoding multi-scale features. 
TABLE II

GRASP DETECTION ACCURACIES ON CORNELL GRASP DATASET FOR DIFFERENT JACCARD INDEXES AND ANGLE THRESHOLD.

\begin{tabular}{|c|c|c|c|c|c|c|c|c|c|c|c|}
\hline \multirow{2}{*}{ Method } & \multirow{2}{*}{ Splitting } & \multicolumn{5}{|c|}{ Jaccard index } & \multicolumn{5}{|c|}{ Angle threshold } \\
\hline & & 0.20 & 0.25 & 0.30 & 0.35 & 0.40 & $30^{\circ}$ & $25^{\circ}$ & $20^{\circ}$ & $15^{\circ}$ & $10^{\circ}$ \\
\hline Song et al. [32] & & - & 95.6 & 94.9 & 91.2 & 87.6 & - & - & - & - & - \\
\hline Chu et al. [33] & $\operatorname{Img}(\%)$ & - & 96.0 & 94.9 & 92.1 & 84.7 & - & - & - & - & - \\
\hline Zhou et al. [26] & & 98.31 & 97.74 & 96.61 & 95.48 & - & 97.74 & 97.74 & 97.18 & 94.35 & 86.44 \\
\hline Ours & & 99.54 & 99.09 & 99.09 & 99.09 & 98.17 & 99.09 & 99.09 & 97.26 & 95.89 & 93.15 \\
\hline Song et al. [32] & & - & 97.1 & 97.1 & 96.4 & 93.4 & - & - & - & - & - \\
\hline Chu et al. [33] & Obj (\%) & - & 96.1 & 92.7 & 87.6 & 82.6 & - & - & - & - & - \\
\hline Zhou et al. [26] & & 97.74 & 96.61 & 93.78 & 91.53 & - & 96.61 & 96.04 & 95.48 & 93.22 & 85.31 \\
\hline Ours & & 98.64 & 98.64 & 97.73 & 96.82 & 95.91 & 98.64 & 98.64 & 98.18 & 97.73 & 94.55 \\
\hline
\end{tabular}

TABLE III

Performance of different algorithms on Cornell Grasp DATASET.

\begin{tabular}{ccccc}
\hline Method & Img $(\%)$ & Obj (\%) & speed (fps) & Input data format \\
\hline Asif et al. [34] & 88.2 & 87.5 & - & RGB \\
Kumra et al. [35] & 89.21 & 88.96 & 16.03 & Point cloud \\
Song et al. [32] & 95.6 & 97.1 & - & RGB \\
Chu et al. $[33]$ & 96.0 & 96.1 & 8.33 & Point cloud \\
Asif et al. [28] & 97.5 & - & 9 & RGB \\
Zhou et al. [26] & 97.74 & 96.61 & 8.51 & RGB \\
\hline \hline Ours & $\mathbf{9 9 . 0 9}$ & $\mathbf{9 8 . 6 4}$ & $\mathbf{6 6 . 7}$ & RGB \\
\hline
\end{tabular}

\section{B. Grasp Detection in Cornell Dataset}

AFFGA-Net is trained and tested in image- and object-wise splitting respectively. The accuracy is evaluated by the metric in Sec. IV-C and the results are shown in Table. III. AFFGANet achieves accuracy of $99.09 \%$ and $98.64 \%$ in imageand object-wise splitting respectively. Moreover, AFFGA-Net completes a grasp detection pipeline within $15 \mathrm{~ms}$ from reading a RGB image to output an OAR-model at every pixel, which can be used for real-time applications.

In Table. III, we compare AFFGA-Net with representative planar grasp detection methods in public Cornell dataset and with the same experiment conditions, i.e. evaluation methods in Sec. IV-A and evaluation metrics in Sec. IV-C. Based on the Cornell dataset, different input data formats are used in different methods: RGB images are used in [34] [32] [28] [26] and our method, point cloud is used in [35] and [33]. The results show that, we obtain the maximal accuracy using less scenarios information. Methods in Table. III use a grasping rectangle to represent gripper configuration and use the most advanced network in the field of target detection to detect rectangles. However, the ground truth of their network are a set of discrete rectangular boxes, which is inconsistent with the actual grasping attribute of the object. Instead, our AGAmodel is pixel-level to cover the grasping attribute of the object and has fewer variables to make the network easy to train.

Asif et al. [28] use a group of upsampling layers to predict grasping rectangles on each pixel. However, pure upsampling layers cannot adapt to objects with different scales. Instead, we use HASP to improve the adaptability of AFFGA-Net to objects with different scales, and use AD to optimize the shape

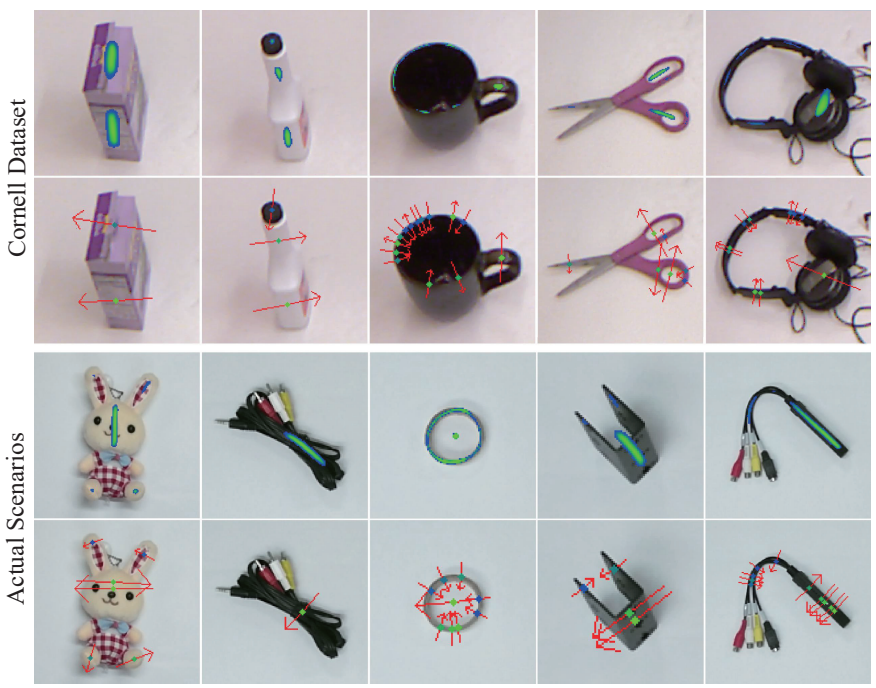

Fig. 6. Grasp detection results on Cornell dataset and actual scenarios. The first row visualizes all the grasp points with grasp confidences over 0.5 , and the grasp points in green have higher grasp confidence. The second row visualizes the OAR-models whose grasp confidences are greater than their 8-connected neighbors.

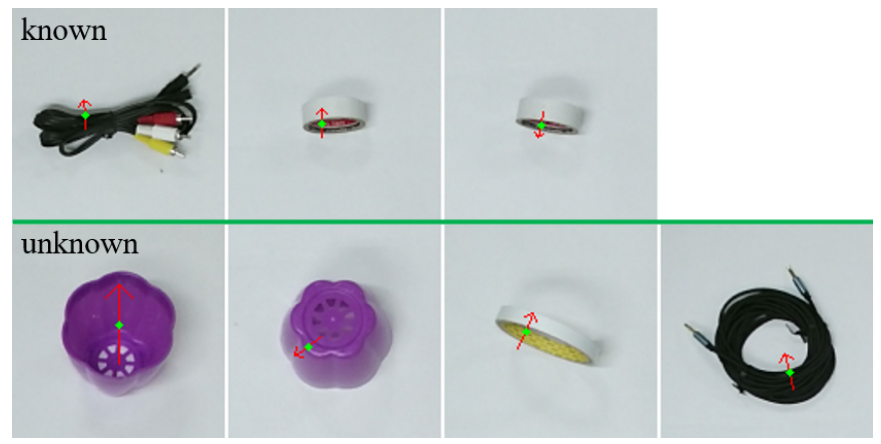

Fig. 7. Unsuccessful detection results of known objects and unknown objects in the actual scenarios. known: Objects in Clutter dataset. unknown: Novel objects.

of the grasp region, which improves the accuracy.

To evaluate robustness, our method is compared with the methods of [32] [33] and [26] in Table. II, under varying Jaccard index and angle threshold. As the threshold increases, the accuracies of [32] [33] [26] decrease rapidly, while our method still has a high accuracy rate. Moreover, our 
TABLE IV

PERFORMANCE OF DIFFERENT ALGORITHMS ON GRASP DETECTION AND ROBOTIC GRASPING WITH UNKNOWN OBJECTS.

\begin{tabular}{|c|c|c|c|c|c|}
\hline Method & Detection accuracy $(\%)$ & $\begin{array}{l}\text { Grasp } \\
\text { Single }\end{array}$ & $\begin{array}{l}\text { ess rate }(\%) \\
\text { Clutter }\end{array}$ & Input data format & Gripper \\
\hline Mahler et al. [7] & - & 80 & - & Point cloud & Parallel-jaw \\
\hline Douglas et al. [36] & - & 92 & 87 & Point cloud & Parallel-jaw \\
\hline Asif et al. [28] & 97.2 & - & 90 & RGB & Parallel-jaw \\
\hline Mahler et al. [10] & - & - & 91.5 & Point cloud & Parallel-jaw \\
\hline Pas et al. [3] & - & - & 93 & Point cloud & Parallel-jaw \\
\hline Wu et al. [18] & - & 95.5 & 93.1 & Point cloud & Parallel-jaw \& Three-fingered \\
\hline Ours & 98.0 & 98.77 & 93.69 & RGB & Parallel-jaw \& Three-fingered \\
\hline
\end{tabular}

Single: The grasp success rate in the scenarios of 1 object. Clutter: The grasp success rate is equal to the average of the success rate in the scenarios of 5 objects and 10 objects.

method achieves the best results in all experiments. There are three reasons that contribute to this achievement. Firstly, the proposed OAR-model avoids the confusion of the neural network learning; Secondly, the AGA-model makes full use of contextual information; Lastly, the proposed AFFGA-Net adaptively generates features for objects with different scales and shapes.

In Fig. 6, we visualize the detection results of some objects. The predicted grasp region covers almost all the graspable positions of the object. Grasp point with maximal confidence tends to appear in the middle of the graspable part, which makes the grasping stable. For objects with small space such as scissor handles and tape, the predicted grasp angle is toward the side with smaller space, or the OAR-model spans the entire ring; hence, the multi-fingered gripper can grasp the object stably. The frequent problem in other methods, that the grasp point with the maximal confidence tends to appear in the centre of all labelled grasp [37], does not appear in our method.

\section{Grasp Detection in Actual Scenarios}

To evaluate the grasp detection performance in real scenarios, we design a grasp detection experiment, and the experimental conditions are setting to be the same as [7]. We sample one object from the Test-set dataset in order and put the object on the center of the workspace in random. Specifically, a human operator samples a random pose of object by shaking the object in a box and placing it upside down in the workspace. On each timestep, the AFFGA-Net receives a RGB image taken by a single overhead depth camera as input and outputs the OAR-model with the highest confidence. The accuracy is evaluated by the metric in Sec. IV-C. We test 10 random orientations of each object.

Our method achieves grasp detection accuracy of $98.50 \%$ with known objects and $98.0 \%$ with unknown objects. The accuracy with unknown objects is shown in Table. IV. Fig. 6 visualizes the detection results of some objects in the actual scenarios. The results show that our method is efficient for objects that have not been trained, even though the category of the object is unknown. We have 7 unsuccessful detections in total, which is shown in Fig. 7. The white tape is recognized as the background due to their similarity, and the pattern inside objects also affects the performance.
TABLE V

ROBOTIC GRASP SUCCESS RATE OF OUR METHOD (\%).

\begin{tabular}{ccccccc}
\hline Gripper & \multicolumn{3}{c}{ Parallel-jaw } & \multicolumn{3}{c}{ Three-fingered } \\
Objects $(M)$ & 1 & 5 & 10 & 1 & 5 & 10 \\
\hline Known & 99.50 & 95.69 & 93.46 & 99.01 & 94.79 & 93.02 \\
Unknown & 98.52 & 95.24 & 93.46 & 99.01 & 93.46 & 92.60 \\
\hline
\end{tabular}

Known: Objects in Clutter dataset. Unknown: Novel objects.

\section{Robotic Grasping}

To study performance on a physical robot, we design a robotic grasping experiment with reference to [7] [10]. The set-up is shown in Fig. 5. First, we sample $M$ objects from the Test-set at random. Then, each of the $M$ objects is randomly placed on the center of the workspace. On each timestep, with our grasping policy, we input a RGB image, point cloud, and camera intrinsics, and output multiple OAR-model candidates with grasp confidences $Q$ over 0.5 (Fig. 8). We select the optimal OAR-model $G r^{*}$ by:

$$
\begin{aligned}
& G r^{*}=\underset{G r}{\arg \max } Q \\
& \text { s.t. }\left\{\begin{array}{l}
D_{x y}-D_{1} \geq D_{t h} \\
D_{x y}-D_{2} \geq D_{t h} \\
Q>0.5
\end{array}\right.
\end{aligned}
$$

where $D_{x y}$ refers to the height of the grasp point relative to the platform, and $D_{1}$ and $D_{2}$ refer to the height of the center of $d_{1}$ and $d_{2}$ in the OAR-model relative to the platform respectively. $D_{t h}$ is set to 0.005 meter in our experiments. Based on the optimal OAR-model $\mathrm{Gr}^{*}$, the robot then approaches the target and closes the jaws. Grasp success is defined by whether or not the grasp transport the target object to the receptacle.

The three-fingered gripper and a parallel-jaw (freeze one finger of the three-fingered gripper) are tested in our experiments. If the robot has 5 consecutive failed grasps, the objects on the workspace are randomly placed again. Each object is successfully grasped 10 times in each set of experiments. If multiple objects are grasped at the same time, one of the objects is randomly selected and put into the receptacle, and the other objects are randomly placed on the workspace again. AFFGA-Net is trained on Cornell and Clutter dataset. 


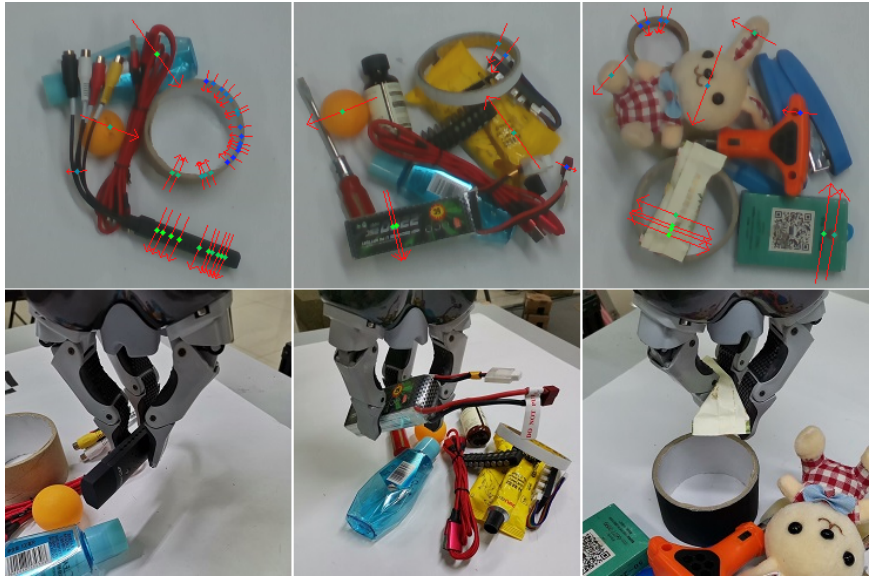

Fig. 8. Experiments for real-world robotic grasping.

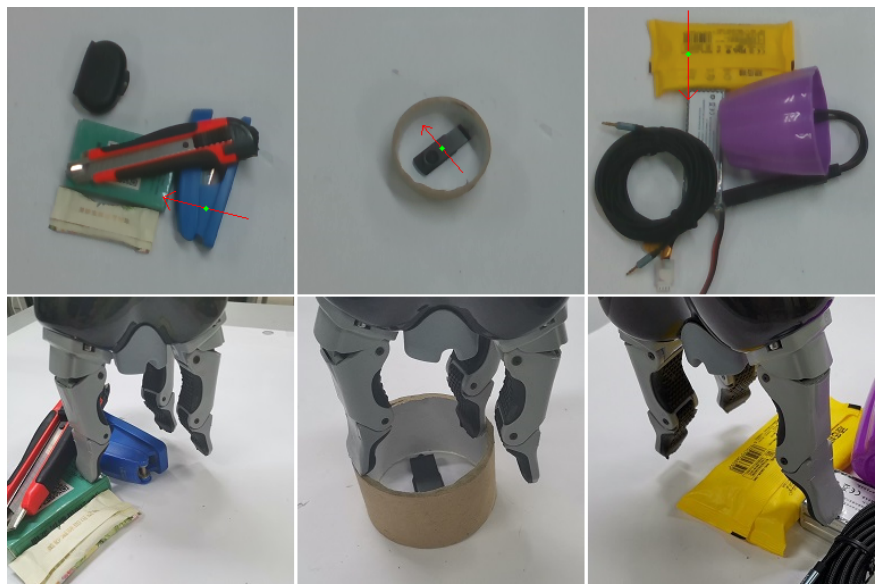

Fig. 9. Three examples of failed grasps. The most common failure modes is that the gripper is blocked by other objects.

Table. $\mathrm{V}$ shows the performance with $M=\{1,5,10\}$ test objects. In order to successfully grasp each object 10 times in each set of experiments, we conduct a total of 2,511 grasping trials. The total number of failed grasps is 111 . There are three main types of failed grasping: (1) The gripper is blocked by other objects when approaching the object (Fig. 9); (2) The object is touched by the gripper and deviates from the original position; (3) The object falls while being lifted. The three-fingered gripper performs better on singleobject scenarios, and the parallel-jaw gripper performs better on cluttered scenarios. On single-object scenarios, the larger grasp range of the three-fingered gripper makes the grasp more stable. On cluttered scenarios, the jaw size of the threefingered gripper is larger, and it is more likely to be blocked by other objects, which causes grasp failure. The experimental procedure is shown in the supplemental video ${ }^{2}$.

In Table. IV, we compare the performance of different algorithms on robotic grasping with unknown objects. The physical set-up used by these researchers are not exactly the same, such as the robot and test objects. Our method achieves grasp success rate of $98.77 \%$ on single-object scenarios and $93.69 \%$

${ }^{2} \mathrm{https}: / /$ youtu.be/ccA1jkkbBJA (a)

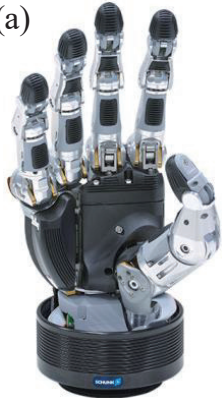

From https://schunk.com/de en gripping-systems/highlights/svh/) (b)

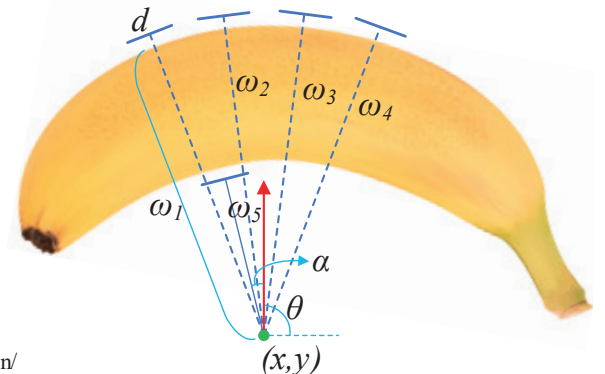

Fig. 10. (a): A multi-finger fully-actuated anthropomorphic hand. (b): A possible improvement of the OAR-model. It is a possible way to introduce five $\omega_{i}$ to present multi-finger instead of one parameter $\omega$ in formula (1), and also introduce a new parameter $\alpha$ to represent the angle of the closed direction of the thumb relative to the axis of the palm. Similar to the original OAR-model, $\theta$ is defined to represent the palm orientation relative to the horizontal axis of the image, and $d$ is the size of each finger. Based on this improved OAR-model for multifinger grasping, the original AGA-model can be extended to multi-finger with more parameters, and the original AFFGA-Net can be modified by designing new Grasping Heads in Fig. 4, making the whole structure adapt to multi-finger grasping.

on cluttered scenarios. Compared with other method based on RGB images [28], our method has a higher grasp success rate in cluttered scenarios. Compared with methods based on point cloud [36] [3] [18], our method avoids collecting large amounts of point clouds to train the network, and avoids learning the gap between simulation and reality [7] [10].

\section{CONCLUSION AND FUtURE WORK}

In this paper, we propose a fast pixel-level grasp detection method. Firstly, a novel Oriented Arrow Representation model (OAR-model) is introduced to represent the full gripper configuration, which is universal to parallel-jaw and three-fingered gripper. In addition, we propose a novel Adaptive Grasping Attribute model (AGA-model) to model the grasping attribute of the object, which resolves angle conflicts in training and avoids the extremely complicated pixel-level labeling process. At last, a new network called Adaptive Feature Fusion and Grasp Aware Network (AFFGA-Net) is proposed to predict the pixel-level OAR-models on RGB images. The pixel-level mapping avoids missing ground-truth grasp postures, and overcomes limitations of current deep-learning grasping techniques by avoiding discrete sampling of grasp candidates and long computation times. Experimental results show that the proposed method achieves the state-of-the-art grasp detection accuracies on Cornell Grasp Dataset and performs well for unknown objects in multi-object stacked scenarios.

Our method has two limitations: (1) The method is only applicative for RGB images and cannot handle point cloud; (2) Two adjacent fingers on the three-fingered gripper are constrained by OAR-model to move synchronously. By separately modeling the closing direction of each finger, it is possible to extend the method in this paper to anthropomorphic hands with more flexibility. Specifically, the modified OAR-model models the closing directions and positions of five fingers of the anthropomorphic hand, as shown in Fig. 10. 


\section{ACKNOWLEDGMENT}

This work is supported in part by National Key R\&D Program of China (NO.2018YFB1305300), National Natural Science Foundation of China (62176138, 62176136), Shandong Provincial Key Research and Development Program (Major Scientific and Technological Innovation Project) (NO.2019JZZY010130, 2020CXGC010207).

\section{REFERENCES}

[1] Y. Hu, X. Wu, P. Geng, and Z. Li, "Evolution strategies learning with variable impedance control for grasping under uncertainty," IEEE Trans. Ind. Electron., vol. 66, no. 10, pp. 7788-7799, 2019.

[2] G. Li, N. Li, F. Chang, and C. Liu, "Adaptive graph convolutional network with adversarial learning for skeleton-based action prediction," IEEE Transactions on Cognitive and Developmental Systems, 2021, doi: 10.1109/TCDS.2021.3103960.

[3] K. S. Andreas ten Pas, Marcus Gualtieri and R. P. Jr., "Grasp pose detection in point clouds," Int. J. Robotics Res., vol. 36, no. 13-14, pp. 1455-1473, 2017.

[4] C. Yang, X. Lan, H. Zhang, and N. Zheng, "Task-oriented grasping in object stacking scenes with crf-based semantic model," in 2019 IEEE/RSJ International Conference on Intelligent Robots and Systems (IROS), pp. 6427-6434, 2019.

[5] A. H. Memar and E. T. Esfahani, "A robot gripper with variable stiffness actuation for enhancing collision safety," IEEE Trans. Ind. Electron., vol. 67, no. 8, pp. 6607-6616, 2020.

[6] Y. Jiang, S. Moseson, and A. Saxena, "Efficient grasping from rgbd images: Learning using a new rectangle representation," in 2011 IEEE International conference on robotics and automation, pp. 3304-3311, 2011.

[7] J. Mahler, J. Liang, S. Niyaz, M. Laskey, R. Doan, X. Liu, J. A. Ojea, and K. Goldberg, "Dex-net 2.0: Deep learning to plan robust grasps with synthetic point clouds and analytic grasp metrics," in Robotics: Science and Systems, 2017.

[8] I. Lenz, H. Lee, and A. Saxena, "Deep learning for detecting robotic grasps," The International Journal of Robotics Research, vol. 34, no. 4-5, pp. 705-724, 2015.

[9] D. Guo, F. Sun, H. Liu, T. Kong, B. Fang, and N. Xi, "A hybrid deep architecture for robotic grasp detection," in 2017 IEEE International Conference on Robotics and Automation (ICRA), pp. 1609-1614, 2017.

[10] M. Jeffrey and G. Ken, "Learning deep policies for robot bin picking by simulating robust grasping sequences," in Conference on robot learning, pp. 515-524, 2017.

[11] J. Mahler, M. Matl, V. Satish, M. Danielczuk, B. DeRose, S. McKinley, and K. Goldberg, "Learning ambidextrous robot grasping policies," Science Robotics, vol. 4, no. 26, 2019.

[12] R. L. Lab, "Cornell grasping dataset," [EB/OL], 2009, http://pr.cs. cornell.edu/grasping/rect_data/data.php Accessed September 1, 2017.

[13] J. Bohg, A. Morales, T. Asfour, and D. Kragic, "Data-driven grasp synthesis-a survey," IEEE Transactions on Robotics, vol. 30, no. 2, pp. 289-309, 2013.

[14] Z. He, C. Wu, S. Zhang, and X. Zhao, "Moment-based 2.5-d visual servoing for textureless planar part grasping," IEEE Trans. Ind. Electron., vol. 66, no. 10, pp. 7821-7830, 2019.

[15] A. Bicchi and V. Kumar, "Robotic grasping and contact: A review," in 2000 IEEE International Conference on Robotics and Automation, pp. 348-353, 2000.

[16] C. Rubert, D. Kappler, A. Morales, S. Schaal, and J. Bohg, "On the relevance of grasp metrics for predicting grasp success," in 2017 IEEE/RSJ International Conference on Intelligent Robots and Systems (IROS), pp. 265-272, 2017.

[17] Y. Inagaki, R. Araki, T. Yamashita, and H. Fujiyoshi, "Detecting layered structures of partially occluded objects for bin picking," in 2019 IEEE/RSJ International Conference on Intelligent Robots and Systems (IROS), pp. 5786-5791, 2019.

[18] B. Wu, I. Akinola, A. Gupta, F. Xu, J. Varley, D. Watkins-Valls, and P. K. Allen, "Generative attention learning: a "general" framework for highperformance multi-fingered grasping in clutter," Auton. Robots, vol. 44, no. 6, pp. 971-990, 2020.

[19] S. R. Lakani, A. J. Rodríguez-Sánchez, and J. H. Piater, "Towards affordance detection for robot manipulation using affordance for parts and parts for affordance," Auton. Robots, vol. 43, no. 5, pp. 1155-1172, 2019.
[20] A. T. Miller, S. Knoop, H. I. Christensen, and P. K. Allen, "Automatic grasp planning using shape primitives," in IEEE International Conference on Robotics and Automation (ICRA), vol. 2, pp. 1824-1829. IEEE, 2003.

[21] J. Mahler, S. Patil, B. Kehoe, J. Van Den Berg, M. Ciocarlie, P. Abbeel, and K. Goldberg, "Gp-gpis-opt: Grasp planning with shape uncertainty using gaussian process implicit surfaces and sequential convex programming," in IEEE International Conference on Robotics and Automation (ICRA), pp. 4919-4926, 2015.

[22] C. Goldfeder, P. K. Allen, C. Lackner, and R. Pelossof, "Grasp planning via decomposition trees," in IEEE International Conference on Robotics and Automation (ICRA), pp. 4679-4684. IEEE, 2007.

[23] A. Saxena, J. Driemeyer, and A. Y. Ng, "Robotic grasping of novel objects using vision," The International Journal of Robotics Research, vol. 27, no. 2, pp. 157-173, 2008.

[24] Q. V. Le, D. Kamm, A. F. Kara, and A. Y. Ng, "Learning to grasp objects with multiple contact points," in 2010 IEEE International Conference on Robotics and Automation, pp. 5062-5069, 2010.

[25] D. Guo, F. Sun, B. Fang, C. Yang, and N. Xi, "Robotic grasping using visual and tactile sensing," Inf. Sci., vol. 417, pp. 274-286, 2017.

[26] X. Zhou, X. Lan, H. Zhang, Z. Tian, Y. Zhang, and N. Zheng, "Fully convolutional grasp detection network with oriented anchor box," in 2018 IEEE/RSJ International Conference on Intelligent Robots and Systems (IROS), pp. 7223-7230, 2018.

[27] S. Ren, K. He, R. Girshick, and J. Sun, "Faster r-cnn: Towards real-time object detection with region proposal networks," in Advances in neural information processing systems, pp. 91-99, 2015.

[28] U. Asif, J. Tang, and S. Harrer, "Densely supervised grasp detector (dsgd)," in Proceedings of the AAAI Conference on Artificial Intelligence, vol. 33, pp. 8085-8093, 2019.

[29] L.-C. Chen, Y. Zhu, G. Papandreou, F. Schroff, and H. Adam, "Encoderdecoder with atrous separable convolution for semantic image segmentation," in Proceedings of the European conference on computer vision (ECCV), pp. 801-818, 2018.

[30] K. He, X. Zhang, S. Ren, and J. Sun, "Deep residual learning for image recognition," in Proceedings of the IEEE conference on computer vision and pattern recognition, pp. 770-778, 2016.

[31] L.-C. Chen, G. Papandreou, I. Kokkinos, K. Murphy, and A. L. Yuille, "Deeplab: Semantic image segmentation with deep convolutional nets, atrous convolution, and fully connected crfs," IEEE transactions on pattern analysis and machine intelligence, vol. 40, no. 4, pp. 834-848, 2017.

[32] Y. Song, L. Gao, X. Li, and W. Shen, "A novel robotic grasp detection method based on region proposal networks," Robotics and ComputerIntegrated Manufacturing, vol. 65, p. 101963, 2020.

[33] F.-J. Chu, R. Xu, and P. A. Vela, "Real-world multi-object, multi-grasp detection," IEEE Robotics and Automation Letters, vol. 3, no. 4, pp. 3355-3362, 2018.

[34] U. Asif, M. Bennamoun, and F. A. Sohel, "Rgb-d object recognition and grasp detection using hierarchical cascaded forests," IEEE Transactions on Robotics, vol. 33, no. 3, pp. 547-564, 2017.

[35] S. Kumra and C. Kanan, "Robotic grasp detection using deep convolutional neural networks," in 2017 IEEE/RSJ International Conference on Intelligent Robots and Systems (IROS), pp. 769-776, 2017.

[36] D. Morrison, P. Corke, and J. Leitner, "Learning robust, real-time, reactive robotic grasping," The International Journal of Robotics Research, vol. 39, no. 2-3, pp. 183-201, 2020.

[37] L. Chen, P. Huang, and Z. Meng, "Convolutional multi-grasp detection using grasp path for rgbd images," Robotics and Autonomous Systems, vol. 113, pp. 94-103, 2019.

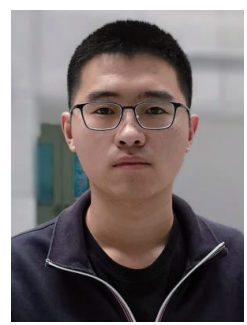

Dexin Wang received his B.S. degree in automation from Shandong University, China, in 2019. Now, he is currently pursuing his M.S. degree in pattern recognition from Shandong University, China. His research interests are computer vision and robotics grasp detection. 


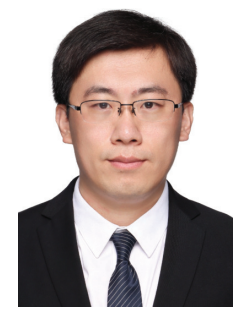

Chunsheng Liu received M.S. degree and Ph.D. degree in Pattern Recognition and $\mathrm{Ma}$ chine Intelligence from Shandong University, Jinan, China in 2012 and 2016, respectively. He was a postdoctor and visiting researcher in University of Washington from 2018 to 2019 . He is currently an associate professor at School of Control Science and Engineering, Shandong University. His research interests include pattern recognition, machine learning, intelligent transportation system, and bionic intelligence.

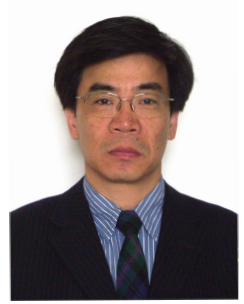

Faliang Chang received his B.S. and M.S. degrees from Shandong Polytechnic University, Jinan, China in 1986 and 1989, respectively. He received his Ph.D. degree in Pattern recognition and Intelligence Systems, Shandong University, in 2003. He has been an professor in Pattern Recognition and Machine Intelligence with the School of Control Science and Engineering, at Shandong University since 2003. Now, his research interests include computer vision, image processing, intelligent transportation system, and multi-camera tracking methodology.

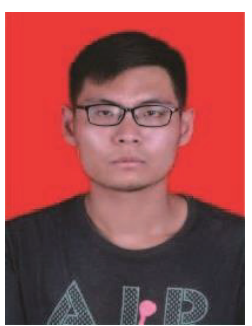

Nanjun Li received his B.S. degree in automation from the North University of China, China, in 2016. Now, he is currently pursuing his Ph.D. degree in pattern recognition from Shandong University, China. His research interests are computer vision and abnormal event detection.

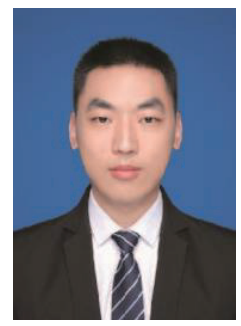

Guangxin $\mathbf{L i}$ received the B.S. degree from Shandong University, Jinan, China, in 2018. He is currently working toward the M.S. degree at the School of Control Science and Engineering, Shandong University, Jinan, China. His research interests include computer vision and action analysis. 